\title{
STATIC AND DYNAMIC STATE ESTIMATION METHOD FOR SOUND ENVIRONMENT BY INTRODUCING FUZZY PROBABILITY
}

\author{
Akira Ikuta*, Mitsuo Ohta** and Yoko NAKASHIMA* \\ * Hiroshima Prefectural Women's University, Japan \\ ikuta@hirojo-u.ac.jp \\ ** Hiroshima University, Emeritus
}

\begin{abstract}
In the measurement of actual sound environment, the observed data show various types of probability distribution forms, and often contain fuzziness due to the existence of confidence limitations in measuring instruments, permissible errors in experimental data, and quantized errors in digitized observation. In this study, by introducing the well-known fuzzy theory, a state estimation method based on the above fuzzy observations is theoretically proposed from both the static and dynamic viewpoints. The validity and the effectiveness of the proposed method are confirmed experimentally by applying it to the evaluation of road traffic noise.
\end{abstract}

\section{INTRODUCTION}

In the actual sound environment, a specific signal shows various types of probability distribution apart from a standard Gaussian distribution due to the diversified causes of fluctuation. Therefore, it is necessary to consider the statistical properties with not only the lower order like mean and variance but also the higher order connected with non-Gaussian properties, in order to estimate several evaluation quantities such as $L_{x}, L_{e q}$, and the peak value, etc. of the specific signal.

On the other hand, the actual observed data often contain fuzziness due to the existence of confidence limitations in measuring instruments, permissible errors in experimental data, and quantized errors in digital observation. Many standard estimation methods proposed previously have not considered positively the fuzziness in the observation data under the restriction of Gaussian type fluctuation, for the simplification of theory [1], [2]. Though several state estimation methods for a stochastic environmental system with non-Gaussian fluctuation have been proposed in our previous studies [3], [4], the fuzziness contained in the observed data has not been considered. Therefore, there arises the problem of how to extend our previous methods to a flexible form applicable also to the ill-conditionedness with fuzzy observation.
In this paper, a new estimation theory for a specific signal, based on the observed data containing the fuzziness and the effects of non-Gaussian properties, is proposed from both the static and dynamic viewpoints. More specifically, by applying fuzzy probability [5] to a probability expression with an infinite expansion series form, a static method to estimate the probability density function of the specific signal based on the fuzzy observation data is first proposed. Next, a dynamical state estimation method of only a specific signal embedded in the additional noise (i.e., background noise) with random fluctuation of non-Gaussian type is theoretically derived in a flexible form applicable to the fuzzy observation data. Finally, the validity and the effectiveness of the proposed method are confirmed experimentally by applying it to the actual road traffic noise data.

\section{STATIC STATE ESTIMATION METHOD BASED ON FUZZY OBSERVATION}

Let us consider the sound environmental system showing an arbitrary fluctuation form of non-Gaussian type. As the probability density function of a specific signal level $X$, the statistical Hermite expansion expression is adopted [6]: 


$$
\begin{aligned}
& P(X)= \frac{1}{\sqrt{2 \pi \sigma_{X}^{2}}} e^{-\frac{\left(X-\mu_{X}\right)^{2}}{2 \sigma_{X}^{2}}}\{1+ \\
&\left.\sum_{n=1}^{\infty} A_{n} \frac{1}{\sqrt{n !}} H_{n}\left(\frac{X-\mu_{X}}{\sigma_{X}}\right)\right\} \\
& \mu_{X}=<X>, \quad \sigma_{X}^{2}=<\left(X-\mu_{X}\right)^{2}>, \\
& A_{n}=<\frac{1}{\sqrt{n !}} H_{n}\left(\frac{X-\mu_{X}}{\sigma_{X}}\right)>
\end{aligned}
$$

On the other hand, the observed data in the actual sound environment often contain fuzziness due to several causes, for example the confidence limitation in measuring instruments, the permissible error of the accuracy in measurements, and the quantized error in the digitization of observation data. After regarding the observed data containing fuzziness as the fuzzy observation $W$, the fuzziness of $W$ is characterized by the following membership function:

$$
\mu_{W}(X)=e^{-\alpha(X-W)^{2}},
$$

where the parameter $\alpha$ can be generally regarded as being unknown. By applying fuzzy probability [5] to Eq.(1), the probability distribution $P(W)$ of fuzzy data $W$ can be expressed as:

$$
P(W)=\frac{1}{K} \int \mu_{W}(X) P(X) d X,
$$

where $K$ is a constant satisfying the normalized condition:

$$
\int P(W) d W=1, \quad\left(\text { or } \sum_{i} P\left(W_{i}\right)=1\right)
$$

In this section, a method to estimate the probability density function $P(X)$ of the specific signal based on the observed fuzzy data $W$ is considered. Substituting Eqs.(1) and (2) into Eq.(3), the following relationship is obtained:

$$
\begin{aligned}
& P(W)=\frac{1}{K} \frac{e^{C_{3}}}{\sqrt{2 C_{1} \sigma_{X}^{2}}} \int \frac{1}{\sqrt{2 \pi \frac{1}{2 C_{1}}}} e^{-\frac{\left(X-C_{2}\right)^{2}}{2 \frac{1}{2 C_{1}}}} \\
& \sum_{n=0}^{\infty} \sum_{r=0}^{n} A_{n} \frac{1}{\sqrt{n !}} d_{n r} H_{r}\left(\frac{X-C_{2}}{\sqrt{1 / 2 C_{1}}}\right) d X \\
& C_{1}=\frac{2 \alpha \sigma_{X}^{2}+1}{2 \sigma_{X}^{2}}, \quad C_{2}=\frac{2 \alpha \sigma_{X}^{2} W+\mu_{X}}{2 \alpha \sigma_{X}^{2}+1},
\end{aligned}
$$

$$
\begin{aligned}
C_{3}= & \frac{2 \alpha \sigma_{X}^{2}+1}{2 \sigma_{X}^{2}}\left\{\left(\frac{2 \alpha \sigma_{X}^{2} W+\mu_{X}}{2 \alpha \sigma_{X}^{2}+1}\right)^{2}\right. \\
& \left.-\frac{2 \alpha \sigma_{X}^{2} W^{2}+\mu_{X}^{2}}{2 \alpha \sigma_{X}^{2}+1}\right\}
\end{aligned}
$$

where $d_{n r}(r=0,1,2, \ldots, n)$ are the expansion coefficients in the equality:

$$
H_{n}\left(\frac{X-\mu_{X}}{\sigma_{X}}\right)=\sum_{r=0}^{n} d_{n r} H_{r}\left(\frac{X-C_{2}}{\sqrt{1 / 2 C_{1}}}\right) .
$$

By considering the orthonormal condition of Hermite polynomial [6]:

$\int \frac{1}{\sqrt{2 \pi \sigma_{X}^{2}}} e^{-\frac{\left(X-\mu_{X}\right)^{2}}{2 \sigma_{X}^{2}}} \frac{1}{\sqrt{n !}} H_{n}\left(\frac{X-\mu_{X}}{\sigma_{X}}\right) \frac{1}{\sqrt{m !}} H_{m}\left(\frac{X-\mu_{X}}{\sigma_{X}}\right) d X=\delta_{n m}$.

Eq.(5) can be expressed as follows:

$$
P(W)=\frac{1}{K} \sum_{n=0}^{\infty} I_{n}\left(W ; \alpha, \mu_{X}, \sigma_{X}^{2}, A_{n}\right),
$$

where

$$
\begin{aligned}
& I_{0}(W ; \bullet)=\frac{e^{C_{3}}}{\sqrt{2 C_{1} \sigma_{X}^{2}}}, \\
& I_{1}(W ; \bullet)=\frac{e^{C_{3}}\left(C_{2}-\mu_{X}\right)}{\sqrt{2 C_{1}} \sigma_{X}^{2}} A_{1} \text {, } \\
& I_{2}(W ; \bullet)=\frac{e^{C_{3}}}{\sqrt{2 C_{1} \sigma_{X}^{2}}} \frac{1}{\sqrt{2 !}}\left\{\frac{\left(C_{2}-\mu_{X}\right)^{2}}{\sigma_{X}^{2}}\right. \\
& \left.+\frac{1}{2 C_{1} \sigma_{X}^{2}}-1\right\} A_{2} \\
& I_{3}(W ; \bullet)=\frac{e^{C_{3}}}{\sqrt{2 C_{1} \sigma_{X}^{2}}} \frac{1}{\sqrt{3 !}}\left\{\frac{3\left(C_{2}-\mu_{X}\right)}{2 C_{1} \sigma_{X}^{3}}\right. \\
& \left.-3\left(\frac{C_{2}-\mu_{X}}{\sigma_{X}}\right)+\frac{\left(C_{2}-\mu_{X}\right)^{3}}{\sigma_{X}^{3}}\right\} A_{3}, \\
& I_{4}(W ; \bullet)=\frac{e^{C_{3}}}{\sqrt{2 C_{1} \sigma_{X}^{2}}}\left\{\frac{9}{\sigma_{X}^{4}}\left(\frac{1}{2 C_{1}}\right)^{2}\right. \\
& +\frac{6\left(C_{2}-\mu_{X}\right)^{2}-6 \sigma_{X}^{2}}{\sigma_{X}^{4}} \frac{1}{2 C_{1}}
\end{aligned}
$$




$$
\left.+\frac{\left(C_{2}-\mu_{X}\right)^{4}-6 \sigma_{X}^{2}\left(C_{2}-\mu_{X}\right)^{2}+3 \sigma_{X}^{4}}{\sigma_{X}^{4}}\right\} A_{4},
$$

Let us assume that $W$ takes the finite numbers of levels $W_{1}, W_{2}, \ldots, W_{L}$, and $W_{i}$ denotes fuzzy data about $W_{i} \mathrm{~dB}$ around the true state value. Therefore, after regarding $\alpha, \mu_{X}, \sigma_{X}$ and $A_{n}(n=1,2, \cdots)$ as unknown parameters, simultaneous equations in the same form as in Eq.(9) can be written by selecting artificially several values of $W_{i}$ with the same numbers as unknown parameters $N(\leq L)$, as follows:

$$
\begin{aligned}
& P\left(W_{1}\right)=\frac{1}{K} \sum_{n=0}^{\infty} I_{n}\left(W_{1} ; \alpha, \mu_{X}, \sigma_{X}^{2}, A_{n}\right), \\
& P\left(W_{2}\right)=\frac{1}{K} \sum_{n=0}^{\infty} I_{n}\left(W_{2} ; \alpha, \mu_{X}, \sigma_{X}^{2}, A_{n}\right), \\
& P\left(W_{N}\right)=\frac{1}{K} \sum_{n=0}^{\infty} I_{n}\left(W_{N} ; \alpha, \mu_{X}, \sigma_{X}^{2}, A_{n}\right) .
\end{aligned}
$$

As a method of solving Eq.(11), the well-known Newton Raphason method can be adopted.

Substituting these estimated parameters in Eq.(1), the probability density function of the true specific signal $X$ eliminating the fuzziness of observation data can be constructed.

\section{DYNAMIC STATE ESTIMATION METHOD BASED ON FUZZY OBSERVATION}

A sound environmental system existing against the background noise of a non-Gaussian distribution is considered. Let the specific signal intensity at a discrete time $k$ be $x_{k}$, and express the dynamical model for the specific signal as:

$$
x_{k+1}=F x_{k}+G u_{k},
$$

where $u_{k}$ denotes the random input with known statistics, and $F$ and $G$ are known system parameters.

The observed data in the actual sound environment often contain fuzziness due to several causes. Therefore, in addition to the inevitable background noise, the effects of the fuzziness contained in the observed data have to be first considered in order to derive a state estimation method for the specific signal. The observation equation can be formulated by dividing it into two types of operation from a functional viewpoint:

1) The additive property of acoustic intensity can be expressed as:

$$
y_{k}=x_{k}+v_{k} .
$$

We assume that the statistics of the background noise intensity $v_{k}$ are known in advance.

2) The fuzzy observation $z_{k}$ is obtained from $y_{k}$. The fuzziness of $z_{k}$ is characterized by the membership function $\mu_{z_{k}}\left(y_{k}\right)$. For example, the following function is adopted as the membership function:

$$
\mu_{z_{k}}\left(y_{k}\right)=\exp \left\{-\alpha\left(y_{k}-z_{k}\right)^{2}\right\}
$$

where $\alpha(>0)$ is a parameter. Though the parameter $\alpha$ in Eq.(14) can be generally given based on prior information (or, through trial and error), it can be regarded as an unknown parameter and is estimated simultaneously with the specific signal $x_{k}$. First, the following simple dynamic model for the parameter is naturally introduced:

$$
\alpha_{k+1}=\alpha_{k} .
$$

In order to derive an estimation algorithm for a specific signal $x_{k}$ based on the fuzzy observation $z_{k}$, we focus our attention on Bayes's theorem for the conditional probability density function. Since the parameter $\alpha_{k}$ is also unknown, the conditional joint probability density function of $x_{k}$ and $\alpha_{k}$ must be considered.

$$
P\left(x_{k}, \alpha_{k} \mid Z_{k}\right)=\frac{P\left(x_{k}, \alpha_{k}, z_{k} \mid Z_{k-1}\right)}{P\left(z_{k} \mid Z_{k-1}\right)},
$$

where $Z_{k}$ is a set of observation data up to time $k$. Based on Eq.(16), through a similar calculation process to that used in a previously reported estimation method [4], the estimate of an arbitrary polynomial function $f_{N_{1}, N_{2}}\left(x_{k}, \alpha_{k}\right)$ of $x_{k}$ and $\alpha_{k}$ of $N\left(=\left(N_{1}, N_{2}\right)\right)$ th order can be derived in an infinite series expression, as follows:

$$
\hat{f}_{N_{1}, N_{2}}\left(x_{k}, \alpha_{k}\right)=<f_{N_{1}, N_{2}}\left(x_{k}, \alpha_{k}\right) \mid Z_{k}>
$$




$$
=\frac{\sum_{l=0}^{N_{1}} \sum_{m=0}^{N_{2}} \sum_{n=0}^{\infty} c_{l m} B_{l m n} \varphi_{n}^{(3)}\left(z_{k}\right)}{\sum_{n=0}^{\infty} B_{00 n} \varphi_{n}^{(3)}\left(z_{k}\right)}
$$

with

$$
B_{\text {lmn }}=<\varphi_{l}^{(1)}\left(x_{k}\right) \varphi_{m}^{(2)}\left(\alpha_{k}\right) \varphi_{n}^{(3)}\left(z_{k}\right) \mid Z_{k-1}>\text {. }
$$

The three functions $\varphi_{l}^{(1)}\left(x_{k}\right), \varphi_{m}^{(2)}\left(\alpha_{k}\right)$ and $\varphi_{n}^{(3)}\left(z_{k}\right)$ are the orthonormal polynomials of degrees $l, m$ and $n$, with weighting functions $P_{0}\left(x_{k} \mid Z_{k-1}\right), \quad P_{0}\left(\alpha_{k} \mid Z_{k-1}\right)$ and $P_{0}\left(y_{k} \mid Z_{k-1}\right)$, which can be artificially chosen as the probability density functions describing the above dominant parts of the actual fluctuation, or as well-known standard probability density functions such as Gaussian or Gamma distribution functions. All the coefficients $c l m$ are appropriate constants when the function $f_{N_{1}, N_{2}}\left(x_{k}, \alpha_{k}\right)$ is expressed in a series expansion form using $\left\{\varphi_{l}^{(1)}\left(x_{k}\right)\right\}$ and $\left\{\varphi_{m}^{(2)}\left(\alpha_{k}\right)\right\}$ :

$$
f_{N_{1}, N_{2}}\left(x_{k}, \alpha_{k}\right)=\sum_{l=0}^{N_{1}} \sum_{m=0}^{N_{2}} c_{l m} \varphi_{l}^{(1)}\left(x_{k}\right) \varphi_{m}^{(2)}\left(\alpha_{k}\right) \text {. }
$$

As a concrete example of a standard probability density function, the well-known Gaussian distribution is adopted:

$$
\begin{aligned}
& P_{0}\left(x_{k} \mid Z_{k-1}\right)=N\left(x_{k} ; x_{k}^{*}, \Gamma_{k}\right), \\
& P_{0}\left(\alpha_{k} \mid Z_{k-1}\right)=N\left(\alpha_{k} ; \alpha_{k}^{*}, \Gamma_{\alpha_{k}}\right), \\
& P_{0}\left(z_{k} \mid Z_{k-1}\right)=N\left(z_{k} ; z_{k}^{*}, \Omega_{k}\right)
\end{aligned}
$$

with

$$
\begin{aligned}
& N\left(x ; \mu, \sigma^{2}\right)=\frac{1}{\sqrt{2 \pi \sigma^{2}}} \exp \left\{-\frac{(x-\mu)^{2}}{2 \sigma^{2}}\right\}, \\
& x_{k}^{*}=<x_{k}\left|Z_{k-1}>, \Gamma_{k}=<\left(x_{k}-x_{k}^{*}\right)^{2}\right| Z_{k-1}>, \\
& \alpha_{k}^{*}=<\alpha_{k}\left|Z_{k-1}>, \quad \Gamma_{\alpha_{k}}=<\left(\alpha_{k}-\alpha_{k}^{*}\right)^{2}\right| Z_{k-1}>, \\
& z_{k}^{*}=<z_{k}\left|Z_{k-1}>, \Omega_{k}=<\left(z_{k}-z_{k}^{*}\right)^{2}\right| Z_{k-1}>. \quad(21)
\end{aligned}
$$

Then, the orthonormal polynomials with three weighting probability density functions in Eq.(20) can be given in terms of the well-known Hermite polynomials [6]:

$$
\begin{aligned}
\varphi_{l}^{(1)}\left(x_{k}\right) & =\frac{1}{\sqrt{l !}} H_{l}\left(\frac{x_{k}-x_{k}^{*}}{\sqrt{\Gamma_{k}}}\right), \\
\varphi_{m}^{(2)}\left(\alpha_{k}\right) & =\frac{1}{\sqrt{m !}} H_{m}\left(\frac{\alpha_{k}-\alpha_{k}^{*}}{\sqrt{\Gamma_{\alpha_{k}}}}\right), \\
\varphi_{n}^{(3)}\left(z_{k}\right) & =\frac{1}{\sqrt{n !}} H_{n}\left(\frac{z_{k}-z_{k}^{*}}{\sqrt{\Omega_{k}}}\right) .
\end{aligned}
$$

By applying the fuzzy moment [5], the two variables $z_{k}^{*}$ and $\Omega_{k}$ in Eq.(21) and the expansion coefficient $B_{r m n}$ are expressed in concrete form, as follows:

$$
\begin{aligned}
z_{k}^{*}= & \frac{<\mu_{z_{k}}\left(y_{k}\right) y_{k} \mid Z_{k-1}>}{<\mu_{z_{k}}\left(y_{k}\right) \mid Z_{k-1}>}, \\
\Omega_{k}= & \frac{<\mu_{z_{k}}\left(y_{k}\right)\left(y_{k}-z_{k}^{*}\right)^{2} \mid Z_{k-1}>}{<\mu_{z_{k}}\left(y_{k}\right) \mid Z_{k-1}>}, \\
B_{r m n}= & <\frac{1}{\sqrt{r !}} H_{r}\left(\frac{x_{k}-x_{k}^{*}}{\sqrt{\Gamma_{k}}}\right) \frac{1}{\sqrt{m !}} H_{m}\left(\frac{\alpha_{k}-\alpha_{k}^{*}}{\sqrt{\Gamma_{\alpha_{k}}}}\right) \\
& \frac{1}{\sqrt{n !}} \mu_{z_{k}}\left(y_{k}\right) H_{n}\left(\frac{y_{k}-z_{k}^{*}}{\sqrt{\Omega_{k}}}\right) \mid Z_{k-1}> \\
& 1<\mu_{z_{k}}\left(y_{k}\right) \mid Z_{k-1}>.
\end{aligned}
$$

\section{APPLICATION TO ROAD TRAFFIC NOISE ENVIRONMENT}

In order to examine the practical usefulness of the proposed state estimation method based on fuzzy observation, the proposed method is applied to the actual sound environment data. Road traffic noise is adopted as an example of a specific signal with a complex fluctuation form. Based on the observed fuzzy data with very roughly quantized level of $5 \mathrm{~dB}$, we have estimated the probability density function of the road traffic noise by applying the static state estimation method in Sec 2 . The parameters in the probability density function of Eq.(1) have been estimated as the following procedure:

i)Estimation of two parameters $\mu_{x}$ and $\sigma_{x}$, and a parameter $\alpha$ in the membership function by use of only the first term (i.e., $n=0$ ) in the right side of Eq.(11). ii)Determination of a constant $K$ by using the normalized condition of Eq.(4) and the estimated parameters in i). iii)Estimation of expansion coefficients $A_{n} \quad(n=1,2, \cdots)$ by solving the simultaneous equations 
considering the higher terms of Eq.(11) after substituting the parameters determined by the above procedures in i) and ii) in Eq.(11).

Figure 1 shows the estimated probability density function for the road traffic noise based on 500 data. Furthermore, a result for the probability density function based on 200 data is shown in Fig.2. In these figures, the observed values based on the fuzzy data with the quantized level of $5 \mathrm{~dB}$ are shown in (O), and the experimental values based on the precisely observed data are shown in ( $\bullet$ ). Furthermore, theoretically estimated probability density functions are shown in (---: when considering only the first term of Eq.(1), -.-: when considering the expansion terms until $n=3$ in Eq.(1), - : when considering the expansion terms until $n=4$ in Eq.(1)). In the case of Fig.2, non-Gaussian property of the fluctuation becomes remarkable. When taking many more expansion terms $A_{n}$ of higher order into consideration, the theoretically estimated curves in Figs.1 and 2 approach the experimentally sampled values for the probability density function of the road traffic noise.

Next, applying the proposed dynamic state estimation method in Sec. 3 to actually observed data quantized and contaminated by background noise, the fluctuation wave form of the specific signal (i.e., road traffic noise) is estimated. The statistics of the specific signal and the background noise used in the experiment are shown in Table 1. By regarding the quantized observation data

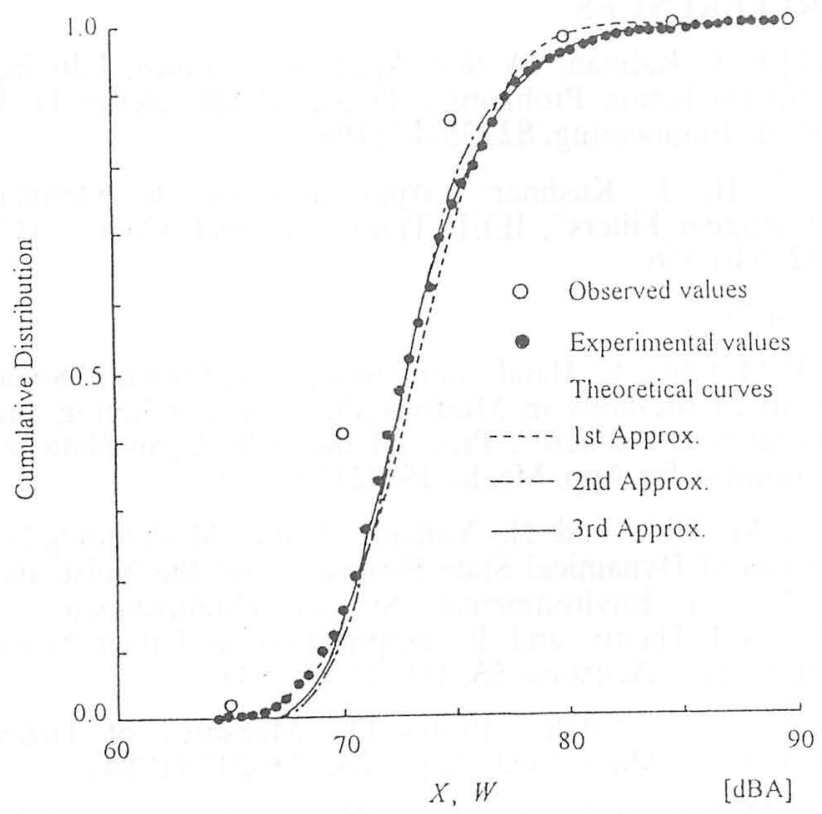

Fig.1 Comparison between theoretically estimated curves and experimentally sampled points on the probability distribution of road traffic noise in the case of 500 data.

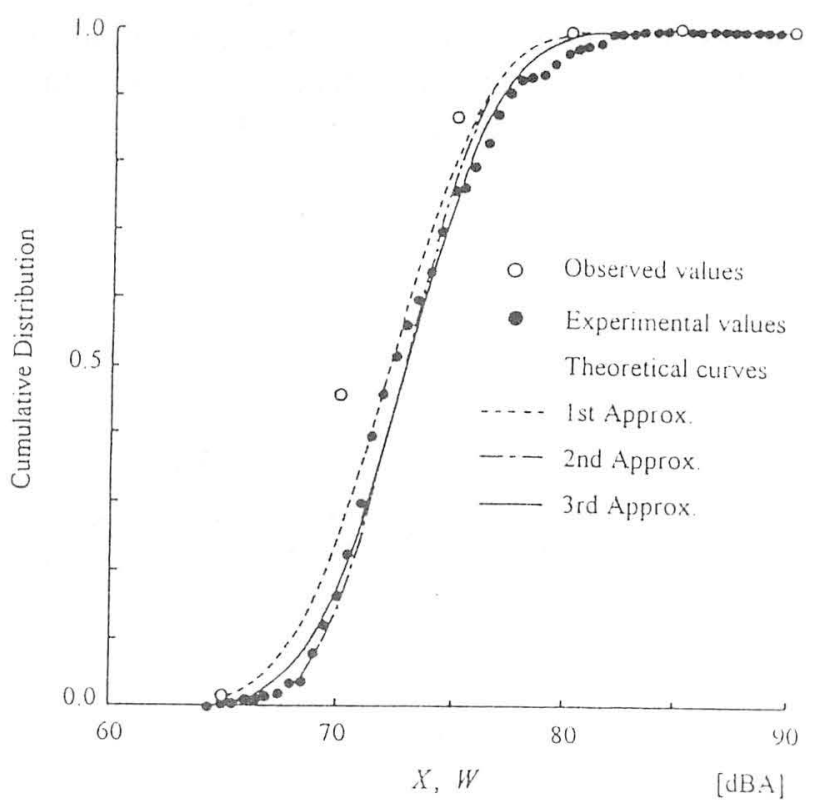

Fig.2 Comparison between theoretically estimated curves and experimentally sampled points on the probability distribution of road traffic noise in the case of 200 data.

Table 1 Statistics of the specific signal and the background noise.

\begin{tabular}{|l|l|l|l|}
\hline \multicolumn{2}{|c|}{ Statistics of specific signal } & \multicolumn{2}{|c|}{ Statistics of background notse } \\
\hline $\begin{array}{l}\text { Mean } \\
\left.\text { Watt } / \mathrm{m}^{2}\right]\end{array}$ & $\begin{array}{l}\text { Standard deviation } \\
{\left[\text { Watt } / \mathrm{m}^{2}\right]}\end{array}$ & $\begin{array}{l}\text { Mean } \\
{\left[\text { Watt } / \mathrm{m}^{2}\right]}\end{array}$ & $\begin{array}{l}\text { Standard deviation } \\
{\left[\text { Watt } / \mathrm{m}^{2}\right]}\end{array}$ \\
\hline $2.9 \times 10^{5}$ & $2.8 \times 10^{5}$ & $2.9 \times 10^{-5}$ & $1 \div \times 10^{-5}$ \\
\hline
\end{tabular}

after contamination by the background noise as fuzzy observation data, the proposed estimation method is applied. Figure 3 shows the estimated results of the fluctuation wave form of the specific signal when the observation data are quantized with $1 \mathrm{~dB}$ width. For comparison, the estimation results calculated using the usual method without considering any membership function are also shown in this figure. Since Kalman's filtering theory is widely used in the field of stochastic system, this method is also applied to the fuzzy observation data as a trial. The results estimated by the proposed method considering the membership function show a more accurate estimation than the results based on the standard type dynamic estimation method (i.e. Kalman filter) without consideration of the membership function.

Figure 4 shows the state estimation results when the roughly quantized data with $2 \mathrm{~dB}$ width are used as fuzzy observation data. The results by the proposed method show good agreement with the true values. On the other hand, there are great discrepancies between the estimates based on the standard method and the true values, particularly in the estimation of the lower level values of the fluctuation. Furthermore, the squared sum 
of the estimation error is calculated in Table 2. From these results, the effectiveness of the proposed method considering the membership function for the fuzzy observation data is confirmed numerically.

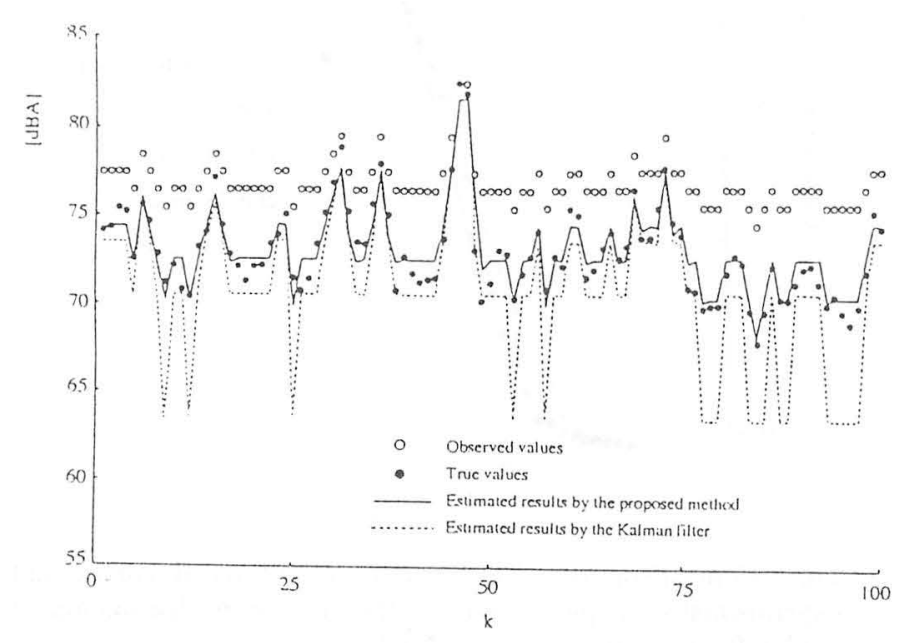

Fig.3 State estimation results for the road traffic noise based on the quantized data with $1 \mathrm{~dB}$ width.

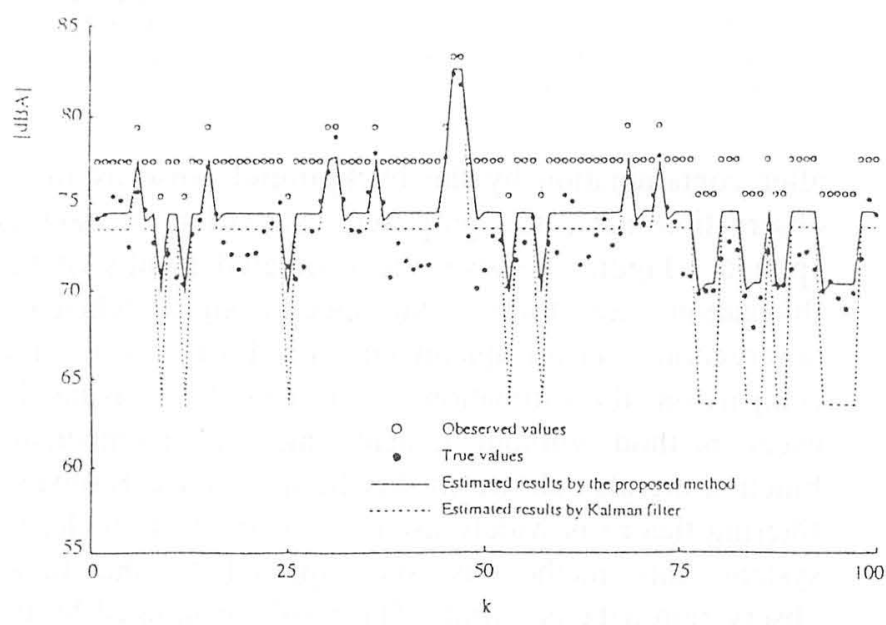

Fig.4 State estimation results for the road traffic noise based on the quantized data with $2 \mathrm{~dB}$ width.

Table 2 Root-mean squared error of the estimation [in $\mathrm{dB}$ ].

\begin{tabular}{|l|c|c|}
\hline $\begin{array}{l}\text { Observed } \\
\text { Data }\end{array}$ & $\begin{array}{l}\text { Proposed } \\
\text { Method }\end{array}$ & $\begin{array}{l}\text { Kalman } \\
\text { Filter }\end{array}$ \\
\hline $\begin{array}{l}\text { Quantized Data } \\
\text { with 1 dB Width }\end{array}$ & 0.90 & 2.52 \\
\hline $\begin{array}{l}\text { Quantized Data } \\
\text { with 2 dB Width }\end{array}$ & 1.72 & 2.65 \\
\hline
\end{tabular}

\section{CONCLUSION}

In this paper, based on the observed data containing fuzziness, a new theory for estimating a specific signal has been proposed especially from two viewpoints of static and dynamic methods. The proposed estimation methods have been realized by introducing a fuzzy theory into the probability density function of expansion series type. The proposed methods have been applied to the actual estimation problem of the sound environment, and it has been experimentally verified that a better result has certainly been obtained than the result employing the usual method without considering any membership function.

The proposed approach is quite different from the traditional standard approach. It is still at an early stage of study, and there remain a number of practical problems to be investigate in the future, starting from the results of the basic study in this paper. Some of the problems are the following: i)The proposed state estimation method should be applied to the other estimation problems, and its practical usefulness should be verified in each actual situation. ii)The proposed theory should further be extended into more actual cases with multi-dimensional state variables and multi-sources for the specific signal.

\section{Acknowledgements}

The authors would like to express their cordial thanks to Messrs. Y. Kobayashi and S. Sakata for their valuable assistance.

\section{REFERENCES}

[1] R. E. Kalman: "A New Approach to Linear Filtering and Prediction Problems", Trans. ASME, Series D, J. Basic Engineering, 82, 35-45 (1960).

[2] H. J. Kushner: "Approximations to Optimal Nonlinear Filters", IEEE Trans. Automat. Contr., AC$12,546-556$

[3] M. Ohta, K. Hatakeyama and M. Nishimura: "Some Unified Methods in Multivariable Linear Filtering and Prediction Problem", Proc. of the 26th Japan National Congress for App. Mech., 199-212 (1984).

[4] M. Ohta and H. Yamada: "New Methodological Trials of Dynamical State Estimation for the Noise and Vibration Environmental System---Establishment of General Theory and Its Application to Urban Noise Problems", Acustica, 55, 199-212 (1984).

[5] L. A. Zadeh: "Probability Measures of Fuzzy Events”, J. Math. Anal. Appl., 23, 199-212 (1984).

[6] M. Ohta and T. Koizumi: "General Treatment of the Response of a Nonlinear Rectifying Device to a Stationary Random Input", IEEE Trans. Inf. Theory, IT14, 595-598 (1968). 